\title{
Bilateral facial paralysis secondary to temporal bone trauma: a case report and literature review
}

\author{
NICOLAU ABRAHAO ${ }^{1}$, Vagner Antonio Rodrigues Silva ${ }^{2}$, Guilherme Guimaraes ${ }^{3}$, and \\ Arthur Castilho ${ }^{4}$ \\ ${ }^{1}$ State University of Campinas \\ ${ }^{2}$ Universidade Estadual de Campinas Faculdade de Ciencias Medicas \\ ${ }^{3}$ Affiliation not available \\ ${ }^{4}$ UNICAMP
}

September 22, 2020

\begin{abstract}
A 23-year-old male that evolved to complete bilateral post-nuclear facial paralysis (House \& Brackman Grade VI) after be involved in automobile accident followed by bilateral temporal bone fracture. The patient undergone a facial decompression surgery after performed an ENoG on the 18th day revealing bilateral absence of muscular response.
\end{abstract}

Bilateral facial paralysis secondary to temporal bone trauma: a case report and literature review

Nicolau Moreira Abrahão*1 (nicolaumabrahao@gmail.com); Guilherma Correa Guimarães $^{1}$ (guilherme.correaguimaraes@gmail.com); Arthur Menino Castilho1(arthurcastilho@gmail.com); Vagner Antônio Rodriguesda Silva ${ }^{1}$ (vagrodrigues@hotmail.com)

${ }^{1}$ Department of Otorhinolaryngology - Rua Tessália Vieira de Camargo, 126 - University of Campinas, Campinas - SP, Brazil

Corresponding Author:

Nicolau Moreira Abrahão

Department of Otorhinolaryngology

School of Medical Sciences - University of Campinas

Cidade Universitária, Campinas, SP, Brazil- 13083-887

Phone: +55 $193521-7454$

$+55193521-7523$

E-mail: nicolaumabrahao@gmail.com

Keywords: Temporal Bone Fracture; Facial Palsy; Facial Nerve Decompression; Wallerian Degenaration

Key Clinical Message

When temporal bone fractures are associated with facial palsy (FP) evolution the medical team have no much time to make a decision: to do or not to do a surgical approach? How to evaluate the neccessity? When to do it and when is the correct time? 


\section{Case report:}

A 23-year-old male involved in a high intensity automobile accident presented at the trauma scene traumatic brain injury (TBI) evolving to right-sided subdural haematoma and immediate bilateral facial paralysis (FP) at the trauma scene. Patient was admitted to Intensive Care Unit and Neurosurgery team opted for a nonsurgical approach. Patient was discharged on the 12th day and referred to ENT team only on the 16th day of FP, reporting bilateral hypoacusis and persistent bilateral facial palsy without improvement. Patient denied dizziness, tinnitus, otorrhagia, otorrhea and rhinorrhea.

The otoscopy examination showed absence of external auditory meatus injuries and intact tympanic membrane associated with bilateral hemotympanum. The VII cranial nerve examination revealed complete bilateral post-nuclear facial paralysis (House \& Brackman Grade VI). All other cranial nerves evaluation was normal. Audiometry demonstrated right mild conductive hearing loss and moderate conductive hearing loss in the left side, with pure tone average (PTA, calculated at $0.5,1,2,4 \mathrm{kHz}$ ) of $35 \mathrm{~dB}$ HL in the right ear and 55 dB HL in the left side. Speech audiometry confirmed left hearing loss.

Maxillofacial computed tomography scan (CT-scan) showed multiple non-displaced fractures lines on the both sides of zygomatic and sphenoid bone and on the posterior wall of left maxillary bone. Temporal CTscan evidenced a longitudinal fracture on the right temporal bone and a transverse fracture on the left side, both sparing the otic capsule (figures 1 and 2).

On the 18th day after trauma, patient performed an electroneuromyography revealing bilateral absence of muscular response to stimulus. Facial nerve decompression was executed and bone spicule were removed bilaterally at the same surgical time (figures 3 and 4). After one-year follow-up, patient presented a House \& Brackman facial palsy grade II bilaterally.

\section{Discussion:}

Bilateral facial nerve paralysis is a rare entity, corresponding to less than $2 \%$ of all facial palsy cases (1). The most important causes are trauma, infectious diseases (e.g. mononucleosis, syphilis, Lyme disease, bilateral otitis media, bilateral Herpes-Zoster), neurological diseases (e.g. multiple sclerosis, stroke and neoplasms) and diseases which remain with uncertain aetiologies, such Guillain-Barre syndrome, Melkersson-Rosenthal syndrome, sarcoidosis, leukaemia (2).

Cranial trauma associated with temporal bone fractures are responsible for $3 \%$ of all facial palsy (FP) cases, which can be traditional divided into longitudinal, transverse and mixed fractures (2). Recently, a new classification was proposed based on the otic capsule, dividing the fractures into otic capsule sparing and otic capsule disrupting (3). The importance to classify the fracture type relies on its prediction of the severity of the signs and symptoms the patient can develop. Longitudinal fractures, usually sparring the otic capsule, corresponds to the majority of all temporal bone fractures and is often associated with mild cases and absence of FP. Transverse fractures are less frequent, but more often associated with otic capsule disruption. Due to the fracture line path goes toward the otic capsule, patients usually present some complications as cerebrospinal fluid fistulae (CSF), otic capsule damage or facial nerve injury (3). The current patient's CTscan evidenced a longitudinal fracture on the right temporal bone and a transverse fracture on the left side, both sparing the otic capsule. As described in the Case Report, the current patient evolved with bilateral FP besides CT-scan showed a pattern of fracture on the right side that it is supposed to predict lower risk of facial nerve injury.

The risk of $\mathrm{FP}$ is 6 to $7 \%$ overall the temporal bone fractures, when $25 \%$ of them are acute, complete and presenting at outset. The remaining $75 \%$ have partial facial nerve palsy at the first evaluation and subsequently worsens within one to 16 days (3) (4) (5). The management of facial paralysis due to temporal bone trauma is a challenging. It is not a simple decision select which patient should go under facial decompression surgery and which should not and might undergo to isolated medical treatment (3).

After any injury to the peripheral nerve the process known as Wallerian degeneration occurs. It is an innate-immune response, culminating in recruitment of macrophages (that will make the phagocytosis of 
degenerated myelin anterogradely to the lesion), and in activation of Schwan cells within the first 24 hours. These cells play an important role in this phenomenon dividing themselves rapidly into two daughter cells and up regulating the gene expression for proteins to assist the degeneration and reparation process (6). The success of the regeneration depends on the degree of nerve injury. In 1943, Seddon described three basic types of injury: neuropraxia, axonotmesis and neurotmesis. In 1951, Sunderland (SD) amplified it classifying injuries into five degrees. Sunderland I is the lightest degree and represents neuropraxia. Sunderland II, III and IV represent different degrees of axonotmesis. Sunderland V represents neurotmesis, being the most severe among all. Highers degrees of SD are associated with more severe facial palsy presentations (3) (6).

The correct therapeutic decision relies on, through clinical evaluation, separate patients in two groups. First group includes patients who have acute, complete facial paralysis at onset, usually at the trauma scene. Normally this group has a higher degree of SD and a potentially fully unrecoverable injury (3). Second group comprises patients who have a delayed facial paralysis onset, commonly associated with a less severe degree of dysfunction and greater capacity to recover. Both groups should be treated within two weeks of systemic corticosteroid and weekly follow-up. The difference between the two groups is in the follow-up in those two weeks. First group is associated with poor recovery in nerve function, so electroneurography (ENoG) can provide useful neurophysiological information, being useful to follow-up and evaluate response to treatment (3) (6).

ENoG measures the evoked compound muscle action potential of the stimulated facial nerve, being the most useful electrodiagnostic test for prognostic information. It should be done after three days of injury as this is the time to the Wallerian degeneration takes to reach the stylomastoid foramen (6). When there is more than $90 \%$ of degeneration within six days or a degeneration higher than $95 \%$ within 14 days, a potentially unrecoverable SD V degree injury may have set, with a poor long-term prognosis. Facial nerve exploration and decompression are usually recommended in this group of patients (3). The timing for facial nerve decompression after the onset still controversial, varying from the first three days to after three weeks of palsy (3). Delayed surgical decompression was generally considered ineffective as a treatment for VII paralysis due to course of Wallerian Degeneration total reach (7). However some authors like Jim Kim et al (8) demonstrated that patients previously treated with steroids since VII paralysis has set out and undergone surgery after three weeks have improved outcomes (8) (9). The majority of temporal bone trauma cases have others life threatening injuries that may delay early surgical decompression but when it is possible is preferable to do within two weeks although the improved outcomes of delayed intervention (8) (9). The current patient had not been treated with corticosteroids and on the $18^{\text {th }}$ day an ENoG was performed revealing bilateral absence of muscular response to stimulus undergoing bilateral surgery decompression at the same day. After one-year follow-up, patient presented a House \& Brackman facial palsy grade II bilaterally.

Acquired CSF leaks in a context of temporal bone trauma occurs approximately $20 \%$ of time and are among the worst complications due to the increased risk of developing meningitis (3). The CSF leaks from the Central Nervous System (CNS) to tympanic cavity, antrum and mastoid air cells cavity through the line fracture in temporal bone and it can present as a clear otorrhea if the tympanic membrane is disrupted or as a clear rhinorrhea if the tympanic membrane is intact (3). Both of them can be present at the onset and If CSF leaks is suspected it must be confirmed using protein electrophoresis to detect $\beta 2$ transferrin - with high sensitivity and specificity - or detecting $\beta$-trace protein in CSF otorrhea or rhinorrhea through the nephelometric analysis demonstrating $100 \%$ positive and negative predictive values in CSF leak detection (3) (10).

The CSF are usually managed conservatively for seven to 10 days with a high rate of success using strategies to minimize the CSF pressure include bed rest with elevation of the head of the bed, stool softeners, instructions to avoid nose blowing, sneezing, and straining, and repeat lumbar punctures or lumbar drain if the leak persists. Because of the increased risk of meningitis following persistent CSF fistulae, surgical closure of fistulae persisting greater than seven to 10 days is recommended (3).

When otic capsula damage occurs severe to profound sensorineural hearing loss (SNHL) install immediatelly 
(3). Conductive Hearing losses (CHL) are caused by hemotympanum, efusion in the middle ear or disruption of the ossicular chaim and are most common in otic capsule disruption (3). The current patient developed hemotympanum as a cause of CHL and did not develop other causes of CHL and SNHL and did not develop CSF leak.

\section{Conclusion:}

Peripheral facial palsy is more associated with transverse temporal bone fracture or, according to the most recent classification, fractures with otic capsule disruption. Medical management begins usually with corticosteroid to prevent inflammatory local reaction and reduce the Wallerian degeneration degree. There is no consensus regarding surgical treatment, however the authors opinion is to evaluate the necessity of facial nerve decompression guided by the clinical characteristics and ENoG result. ENoG should be done after the third day (the authors suggest after the seventh day). If the degree of facial nerve degeneration is more than $90 \%$ within six days or a degeneration higher than $95 \%$ within 14 days facial nerve exploration and decompression are usually recommended.

\section{AUTHOR CONTRIBUTION}

Nicolau Moreira Abrahão: Conception, organization, writing of the manuscript.

Guilherme Correa Guimarães: writing of the manuscript

Arthur Menino Castilho: Review of the manuscript

Vágner Antônio Rodrigues da Silva: Conception, organization, review.

Conflicts of interest: nothing to declare

Acknowledgments:

No other person has contributed to the current manuscript.

There is no financial or material supports.

\section{References:}

1. Pothiawala S, Lateef F. Bilateral Facial Nerve Palsy: A Diagnostic Dilemma. Case Rep Emerg Med. 2012;2012:1-3.

2. Eliçora SŞ, Dinç AE, Bişkin S, Damar M, Bilgin E. Bilateral Facial Paralysis Caused by Bilateral Temporal Bone Fracture: A Case Report and a Literature Review. Case Rep Otolaryngol. 2015;2015:1-4.

3. Diaz R, Cervenka B, Brodie H. Treatment of Temporal Bone Fractures. J Neurol Surg Part B Skull Base. 2 de junho de 2016;77(05):419-29.

4. Brodie HA, Thompson TC. Management of complications from 820 temporal bone fractures. Am J Otol. março de 1997;18(2):188-97.

5. Exadaktylos AK, Sclabas GM, Nuyens M, Schröder D, Gallitz B, Henning P, et al. The Clinical Correlation of Temporal Bone Fractures and Spiral Computed Tomographic Scan: A Prospective and Consecutive Study at a Level I Trauma Center: J Trauma Inj Infect Crit Care. outubro de 2003;55(4):704-6.

6. Lee D-H. Clinical Efficacy of Electroneurography in Acute Facial Paralysis. J Audiol Otol. 2016;20(1):8.

7. Ugo Fisch, Erlo Esslen. Total Intratemporal Exposure of the Facial Nerve Pathologic Findings in Bell's Palsy. Arch Otolaryngol. abril de 1972;95:335-41.

8. Kim J, Moon IS, Lee W-S. Effect of delayed decompression after early steroid treatment on facial function of patients with facial paralysis. Acta Otolaryngol (Stockh). janeiro de 2010;130(1):179-84. 
9. Siang PG, Ying XT, Dayang Suhana AM, Ing PT. Surgical outcomes of transmastoid facial nerve decompression: Preliminary data from a Malaysian tertiary hospital from 2013-2018. Med J Malaysia. 2020;75(3):281-5.

10. Sampaio MH, de Barros-Mazon S, Sakano E, Chone CT. Predictability of quantification of beta-trace protein for diagnosis of cerebrospinal fluid leak: Cutoff determination in nasal fluids with two control groups. Am J Rhinol Allergy. $1^{\circ}$ de novembro de 2009;23(6):585-90.

Figure 1: The CT-Scan of rigth side of temoral bone showed bilateral fracture

Figure 2: The CT-Scan of left side of temoral bone showed bilateral fracture

Figure 3: The transoperative view of left fracture

Figure 4: The transoperative view of rigth fracture

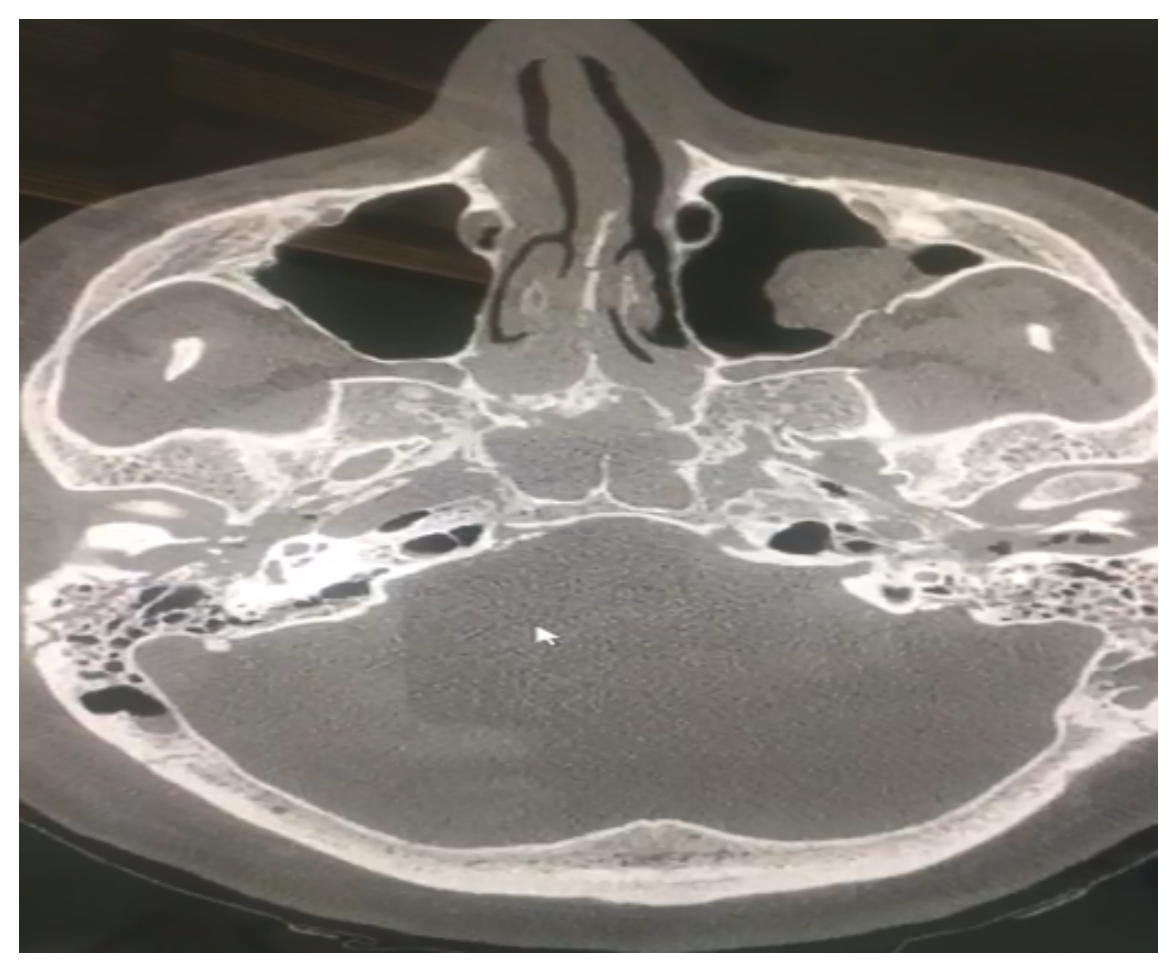



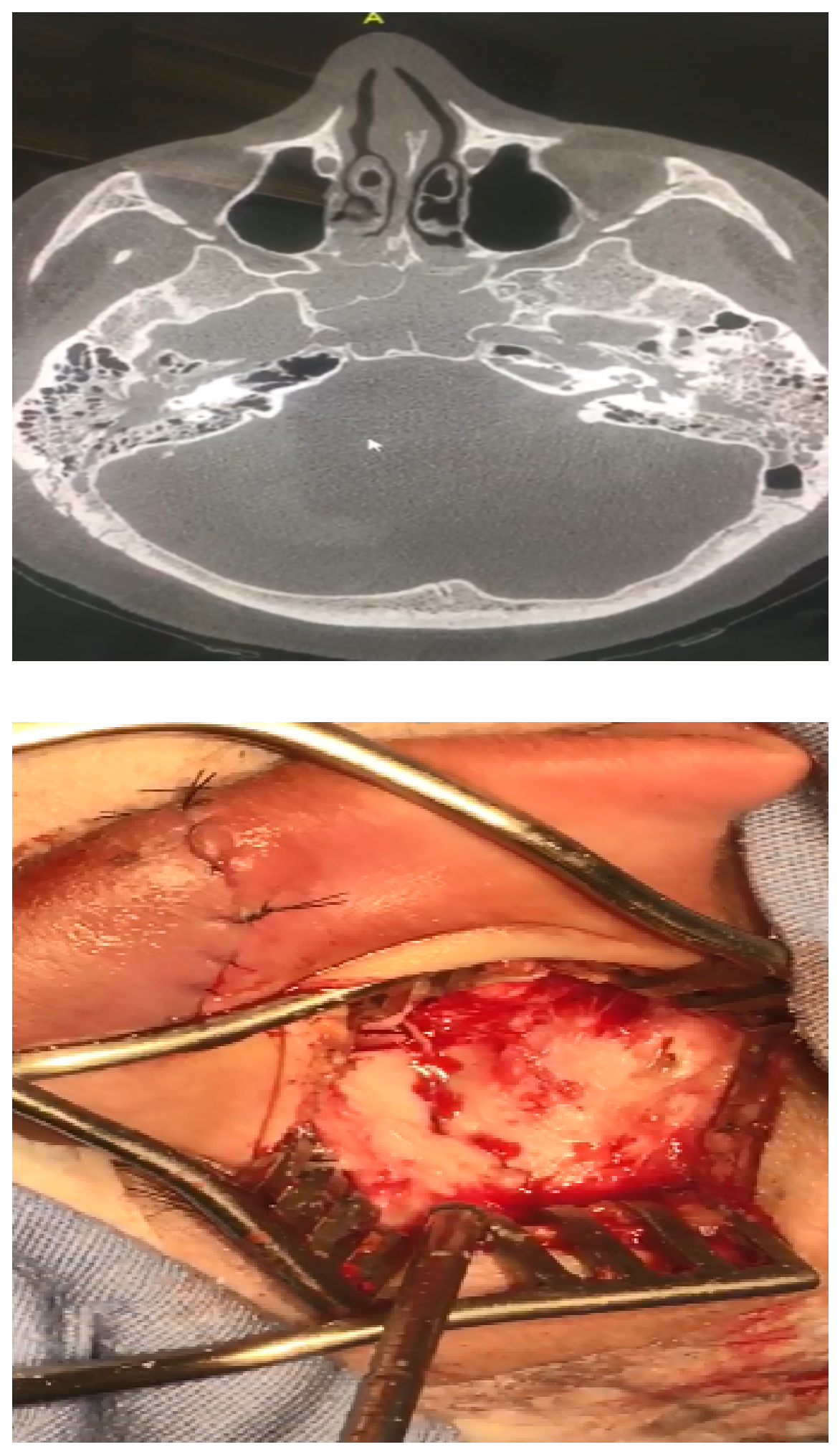


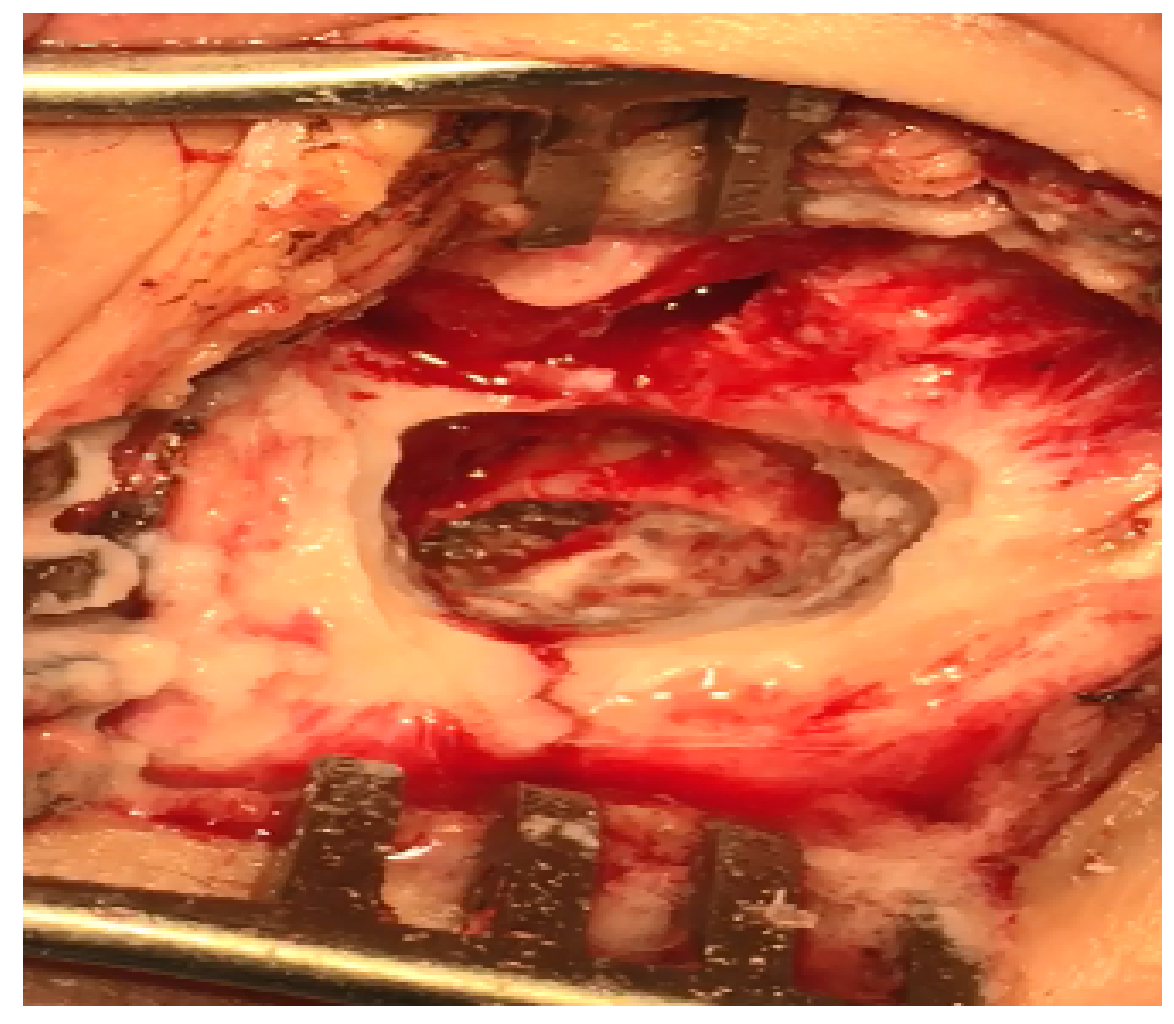

